\title{
First Steps into Practical Engineering for Freshman Students Using MATLAB and LEGO Mindstorms Robots
}

\begin{abstract}
A. Behrens, L. Atorf, R. Schwann, J. Ballé, T. Herold, A. Telle
Besides lectures on basic theoretical topics, contemporary teaching and learning concepts for first semester students give more and more consideration to practically motivated courses. In this context, a new first-year introductory course in practical engineering has been established in the first semester curriculum of Electrical Engineering at RWTH Aachen University, Germany. Based on a threefold learning concept, programming skills in MATLAB are taught to 309 students within a full-time block course laboratory. The students are encouraged to transfer known mathematical basics to program algorithms and real-world applications performed by 100 LEGO Mindstorms robots. A new MATLAB toolbox and twofold project tasks have been developed for this purpose by a small team of supervisors. The students are supervised by over 60 tutors at 23 institutes, and are encouraged to create their own robotics applications. We describe how the laboratory motivates the students to act and think like engineers and to solve real-world issues with limited resources. The evaluation results show that the proposed practical course concept successfully boosts students' motivation, advances their programming skills, and encourages the peer learning process.
\end{abstract}

Keywords: First semester students, freshman course, practical engineering, introduction to programming, MATLAB, LEGO Mindstorms, robotics laboratory, project-based learning.

\section{Introduction}

Practical first semester undergraduate courses in electrical engineering have become more important in modern learning concepts, since they can strongly boost the motivation of first-year students and give insights into practical methods and basic engineering concepts [2]. Against this background and within the scope of the new Bachelor of Science curriculum, instituted by the European Bologna process [1], the Faculty of Electrical Engineering and Information Technology at RWTH Aachen University, Germany established the first semester student laboratory "MATLAB meets LEGO Mindstorms" in the 2007-2008 academic year. The lab focuses on three elements: mathematical methods and digital signal processing fundamentals, programming basics of the MATLAB ${ }^{\circledR}$ software from MathWorks [7], and practical engineering with LEGO ${ }^{\circledR}$ Mindstorms ${ }^{\circledR}$ NXT robots [8].

Freshman introduction courses in practical engineering overcome the traditional twofold teaching structure, which first presents theoretical foundations and only later confronts the more mature student with real-world issues and engineering approaches. This curriculum usually provides only low student motivation and thus the students can encounter difficulties in handling complex situations in their following career [3]. To acquire a better learning process, the essential element can be stated as "no matter how many concepts you teach, no matter how deep you go, no matter how tough your exams are, the only lessons that will remain in the students minds are those that touch them" [4]. Thus it is very important to motivate first semester students by confronting them with practical situations, in which they "feel like engineers" right from the beginning of their studies [2].

In addition to mathematical and electrical engineering skills, the ability to work with computer programming software is also a required skill for today's electrical engineers [5]. For this purpose, a new self-motivated first semester practical laboratory, called "MATLAB meets LEGO Mindstorms", has been developed.

The paper is organized as follows: Section 2 describes the learning targets of the project and the applied software and hardware. The project structure and management executed by a small core team of supervisors is presented in section 3 . Section 4 introduces a novel software toolbox, which has been developed in MATLAB. The students' practical activities, separated into basic exercises and major tasks, are described in section 5. Evaluation results are given in section 6 . In section 7 , some future perspectives are presented, and, section 8 highlights the main conclusions about this practical course.

\section{Learning targets}

The undergraduate laboratory "MATLAB meets LEGO Mindstorms" is motivated by three objectives: mathematical methods, MATLAB, and practical engineering, as illustrated in Fig. 1.

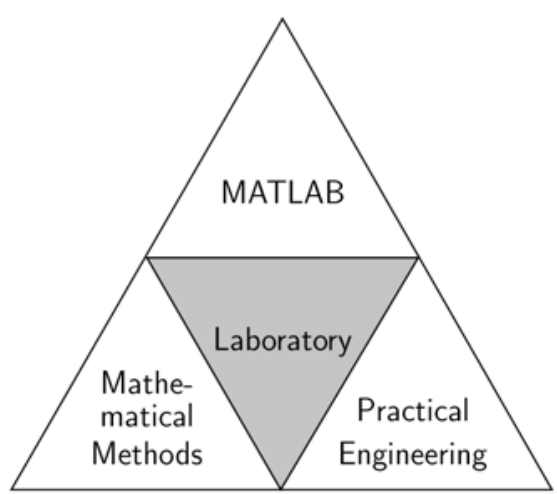

Fig. 1: Key objectives of the laboratory 
The first learning target is characterized by applying and broadening the student's mathematical basic knowledge that has been imparted by the first semester lecture in "Mathematical Methods in Electrical Engineering". The content of this course includes an introduction to digital signal processing and system theory [6] with topics like complex numbers, sampling of functions, the Fourier transform, polynomials, vector spaces and matrix operations. Based on these theoretical foundations, the students are inspired to link theoretical methods to practical issues provided by the last two objectives of the project.

The second objective represents the acquisition of programming skills in MATLAB and the transfer of mathematical methods to programming algorithms. Experience has shown that the most efficient learning process can be achieved by unassisted writing of program code. Therefore the students are encouraged to develop their own code right from the beginning. The introduction of MATLAB as a text-based programming software in this lab is motivated by direct and intuitive access to vectors and matrices. However this software is also widely used in industry and in other courses at RWTH Aachen University.

The third learning target promotes practical engineering of real-world applications and challenging tasks by using programmable and remote-controlled LEGO Mindstorms NXT robots [8]. The standard NXT education kit provides four different sensors (touch, sound, light and ultrasonic, which is used for measuring distances) and motors, illustrated in Fig. 2.

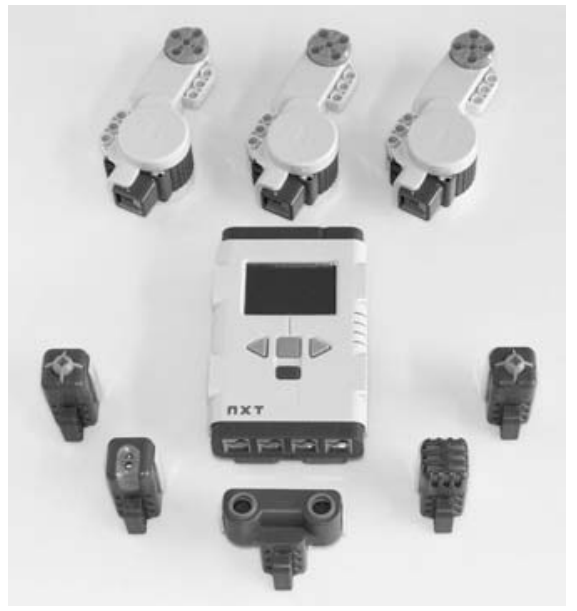

Fig. 2: LEGO Mindstorms NXT hardware

The advantage of using this robotic system is that, on the one hand, complex robots can be easily built with standard LEGO bricks, while on the other hand application constraints are given by the limited brick types and the low-budget sensor accuracy. Limited project resources are essential for simulating authentic engineering tasks and real-world problems.

In addition, the lab supports a peer learning process, in which the students acquire soft skills like collaboration, team work and leadership [5,10].

Overall, the laboratory assists freshmen to handle practical issues in the manner of engineers. The student groups have to build Mindstorms robots and develop algorithms in
MATLAB. Thus, the name of the project is derived straightforward as "MATLAB meets LEGO Mindstorms".

\section{Project structure and management}

The project first took place in December 2007. It was performed as an eight-day full-time (six hours per day) block course. Thus, the students could focus entirely on the project while other first semester lectures paused. 309 freshmen participated in the lab and were guided by more than 60 supervisors at 23 institutes of the Faculty. A total of more than 150 computer workplaces were provided and 100 LEGO Mindstorms NXT robotics sets were bought. Each student group of four shared one robot kit and two computers.

The project management and development of the lab exercises was organized by a small core team of supervisors, who are members of five different departments of the Faculty. Within a four-month period the team determined the required resources, developed a system environment, created lab exercises and demonstrations, and trained 60 supervisors to achieve the proposed learning targets.

Since the focus of the lab is on mathematical methods, digital signal processing, and mechatronic aspects using MATLAB, the NXT robots have to be controlled directly via MATLAB. To provide remote-control and mobility for autonomous robots the usage ofthe wireless Bluetooth ${ }^{\circledR}$ communication interface of the novel LEGO Mindstorms NXT series with its original firmware was chosen. Based on the communication protocol, the core team developed a new MATLAB toolbox, which provides a direct interface between MATLAB and NXT robots. In three-weekly meetings, the software design was defined and the contents of the new lab exercises were discussed and developed. Eventually the practical exercises were documented and introduced to the other supervisors of the Faculty.

\section{RWTH - Mindstorms NXT Toolbox}

Despite the wide choice of commercial and free NXT software for different programming languages [11], no implementation is applicable for the desired direct computer-robot communication via MATLAB without additional software. For this reason, a novel software toolbox, called "RWTH Mindstorms NXT Toolbox" was developed and the LEGO Mindstorms Bluetooth communication protocol [9] were adapted to MATLAB functions. With these functions, all NXT standard sensors and servo motors can be controlled, and various other system features like reading the battery level or playing a tone are implemented. Besides these direct commands, the toolbox is organized in a structure of four command layers, as shown in Fig. 3. It provides additional

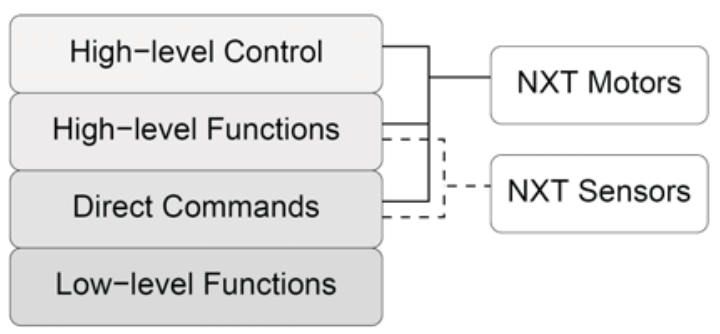

Fig. 3: RWTH - Mindstorms NXT Toolbox - command layers 
high-level and control functions for more intuitive and comfortable usability.

Towards this end the low-level functions and direct commands are encapsulated, and the user interface is adapted to the common MATLAB toolbox usage handling. Fig. 4 shows an example for reading the current measurement value of the ultrasonic sensor. These high-level functions avoid time-consuming programming, especially for beginners. In addition, detailed documentation and program examples are embedded in the toolbox.

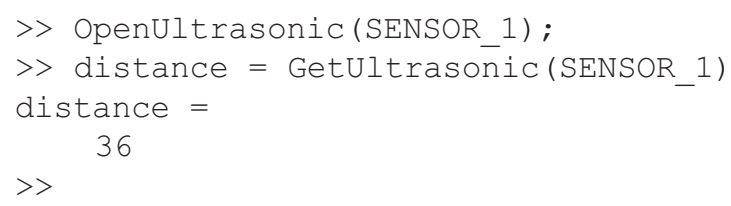

Fig. 4: MATLAB example code using high-level functions for sensor reading

The remote-control concept reduces the implementation of autonomous robots to three main steps: Constructing a robot, developing a MATLAB program, and interactive program execution. There is no need for an intermediate step in which the program is compiled and downloaded onto the embedded robot processor. Therefore a direct debugging and monitoring mode is supported, which allows the students to validate their program code simultaneously with processing. A drawback of this design is related to the characteristics of the wireless Bluetooth interface. In general, the data rate of Bluetooth is limited to 1-3 Mbit/s and data errors and packet losses can occur randomly during the data transmission. However, the major bottleneck is caused by the Bluecore ${ }^{\mathrm{TM}}$ chip of the NXT hardware, which penalizes switching from receive-mode to transmit-mode with an additional $30 \mathrm{~ms}$ latency [9]. Due to these constraints, real-time regulation with a short response time is not possible via the NXT Bluetooth communication protocol.

The RWTH - Mindstorms NXT Toolbox for MATLAB supports Windows and Linux platforms and is published as a free open source product. It is subjected to the GNU General Public License (GPL) [13] and can be downloaded from [14]. In general, the open source software concept motivates people to participate in project development and founds international interacting communities [12]. In addition, the freeware toolbox enables other universities and schools to introduce the software to their practical robotics courses for free and without any obligation.

\section{Practical activities}

The core team has developed nine practical activities, which were performed by the students in two project parts. The first part included six sequential basic exercises, each of which addressed different NXT sensors or actuators. These were developed to teach the students, formed into teams of two, programming principles in MATLAB, applying the RWTH - Mindstorms NXT Toolbox and the Bluetooth interface, and measuring the NXT hardware characteristics.

In the second part of the lab the student groups could choose from three major and more complex tasks, but were also free to develop their own experiments and challenges in order to enhance creative ideas, robot construction and individual problem solving methods. Therefore student groups of four had to develop individual robots and algorithms to solve the major tasks in competition against other groups. In this process the limitations of the LEGO Mindstorms hardware, e.g., slight variations between sensors and motors of the same type, had to be taken into account. Not every behavior of the robot was easily foreseeable in all situations. For instance, the driving distance covered by a robot vehicle showed variations, since it depends on the traction on tiling or on carpet, and had to be considered in the robot design and the control algorithms. It was also necessary to handle the limitations of the Bluetooth channel, which were noticeable in random transmission delays.

All given tasks were designed by the core team with respect to the hardware and Bluetooth constraints, documented and organized in modular subtasks to establish clear requirements and targets for each subtask. At the end of the lab, each student group presented individual robots and algorithms to the other participants. An overview of the lab time schedule is given in Fig. 5.

\begin{tabular}{|c|c|c|c|c|c|c|c|}
\hline 1st & 2nd & 3rd & 4 th & 5th & 6th & 7th & 8th \\
\hline 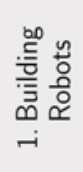 & \multirow{2}{*}{ 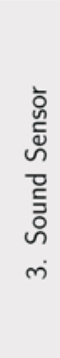 } & \multirow{2}{*}{$\begin{array}{l}\frac{n}{2} \\
\stackrel{0}{0} \\
\dot{\sigma}\end{array}$} & \multirow{2}{*}{ 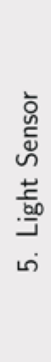 } & \multirow{2}{*}{ 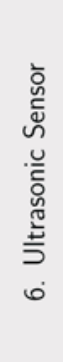 } & \multirow{2}{*}{ 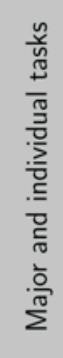 } & \multirow{2}{*}{ 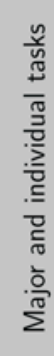 } & 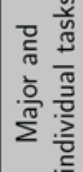 \\
\hline 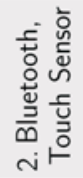 & & & & & & & 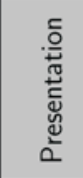 \\
\hline
\end{tabular}

Fig. 5: Lab Time Schedule (in days)

\subsection{Basic exercises}

Each of the six basic exercises focused on a key aspect of the three learning targets, shown in Fig. 1. In the first experiment the students constructed individual robots and tested the sensors with the NXT firmware. In the next two exercises the Bluetooth interface was introduced, and the touch and sound sensor were controlled via MATLAB using the RWTH - Mindstorms NXT Toolbox. In addition, simple programming structures like loops, if-conditions and functions were taught. The fourth experiment was addressed to the NXT servo motors. After taking measurements of the internal rotation sensor and working with different gear transmission ratios, a machine for visualizing complex numbers was built and programmed. For this, the students had to implement an algorithm which reads two complex numbers, takes a mathematical operation from a user input dialog, calculates the result, plots the complex phases in a diagram and also displays them using the mechanical pointers of the LEGO machine, as shown in Fig. 6.

The fifth experiment focused on light sensor measurements and MATLAB GUI design. Finally, the last basic 

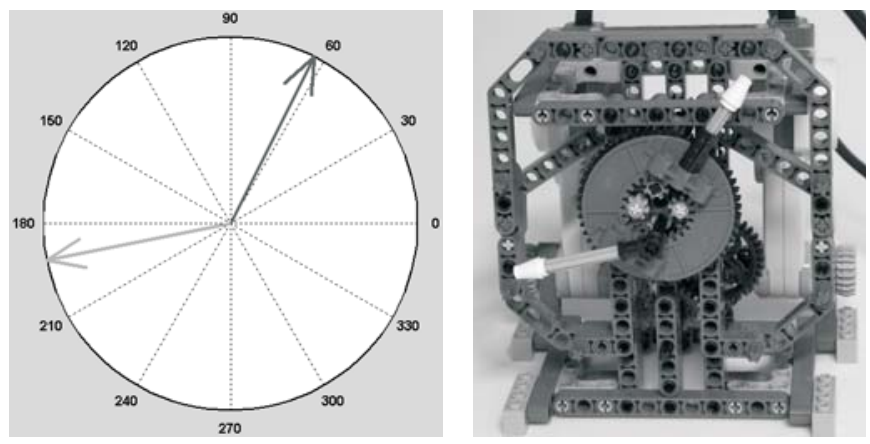

Fig. 6: Exercise 4: Complex Phase Machine (left: MATLAB plot of complex phases, right: Mechanical simulation displayed by the LEGO pointers)

exercise was performed by programming an explorer robot, which scans the local environment using the ultrasonic sensor, plots a 360-degree distance profile and drives autonomously through an open gate of an enclosure, illustrated in Fig. 7. To fulfill this challenge the students had to process the data with simple filter operations, detect signal edges and locate the correct target angle.
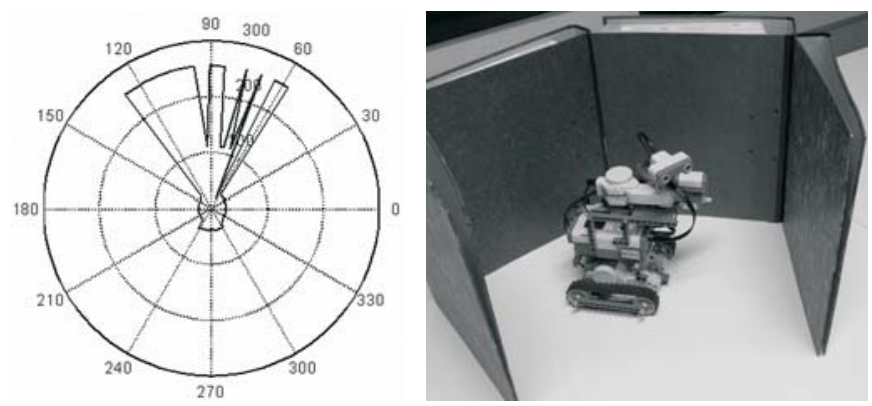

Fig. 7: Exercise 6: Explorer Robot scans the local environment (left: 360-degree distance profile, right: enclosure with an open gate)

\subsection{Major practical tasks}

The major practical tasks were designed to confront the students with more complex challenges, give them the opportunity to work together as a team of four, and manage the assigned tasks on their own. The students could choose among three assigned challenges, or were able to define their own creative application. The three documented tasks included an obstacle course robot which exits and maps a maze, a 3-D robot arm that grabs and sorts colored balls (Fig. 8), and a 2-D robot arm which acts as an image scanner. Besides the competition aspects and the individual robot constructions, all tasks focused on data processing and programming with MATLAB, e.g., data visualization, mapping, GUI interaction and graphical display.

The 2-D scanner application was given by a two-joint robot arm. Students who chose this experiment had to develop a time-efficient scan algorithm. Here the dependency between rotations of the two joints and the current grid position of the light sensor had to be taken into account, as illustrated in Fig. 9. Then challenges like transformation of polar and
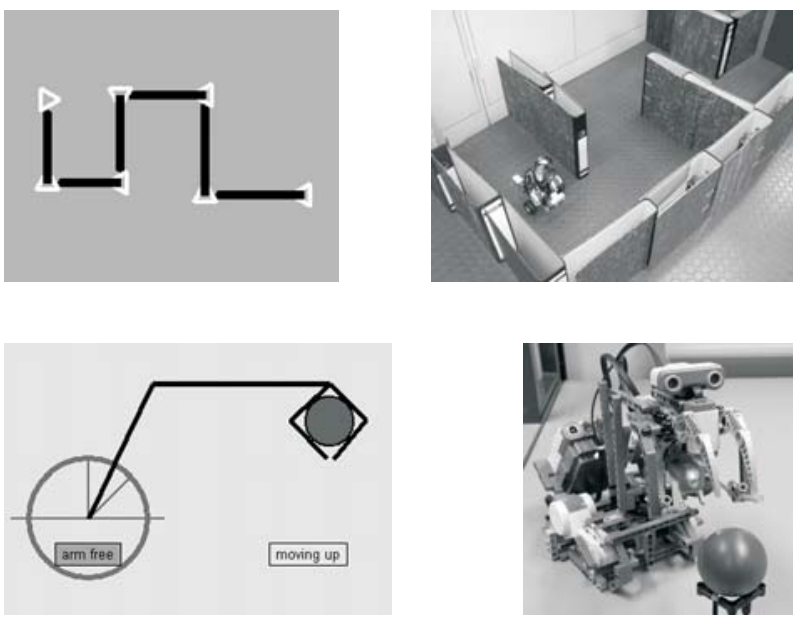

Fig. 8: Major practical tasks (1st row: Obstacle course robot with route mapping, 2nd row: Robot arm with monitored status)

Cartesian coordinates, registration, image interpolation and visualization were addressed in subtasks.

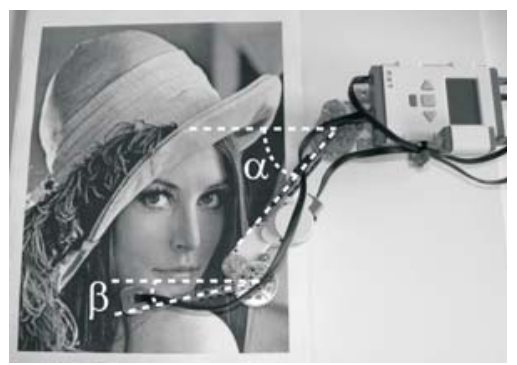

Fig. 9: 2-D Scanner with rotation angles of the joints

The scanning process is illustrated in Fig. 10. The figure shows the original image, a sampled image and the interpolation result of the test picture, called Lena. Despite the hardware limitations the interpolated image provides a good result.
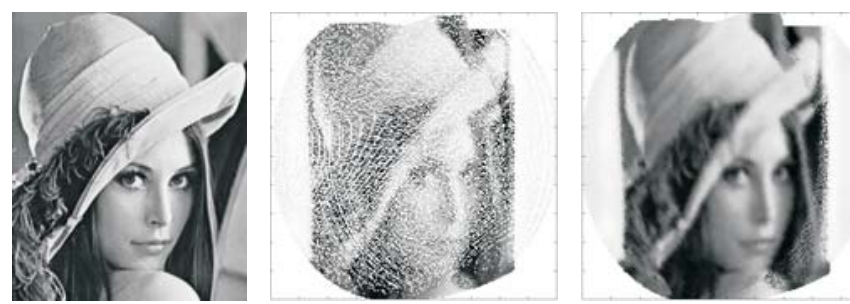

Fig. 10: 2-D Scan (left: original image, middle: sampled image, right: interpolation)

\subsection{Presentation}

On the last day of the lab each student group presented the results and the individual robot constructions to the other participants in a 15-20 minute presentation. The features and behavior of the robots were shown in live demos and the developed MATLAB algorithms were discussed. For the presentation, common software like OpenOffice and Microsoft ${ }^{\circledR}$ PowerPoint was used. 


\section{Evaluation and Results}

After the lab, 175 students participated in an anonymous online evaluation. According to the evaluation results the lab fulfilled the goal of introducing MATLAB as a software for solving real-world problems, based on mathematical methods and principles. As shown in Fig. 11, $48 \%$ of the students rated their MATLAB programming skills as "average" at the beginning of the lab, whereas $48 \%$ evaluated their improved skills as "excellent" and $44 \%$ as "good" after the project.

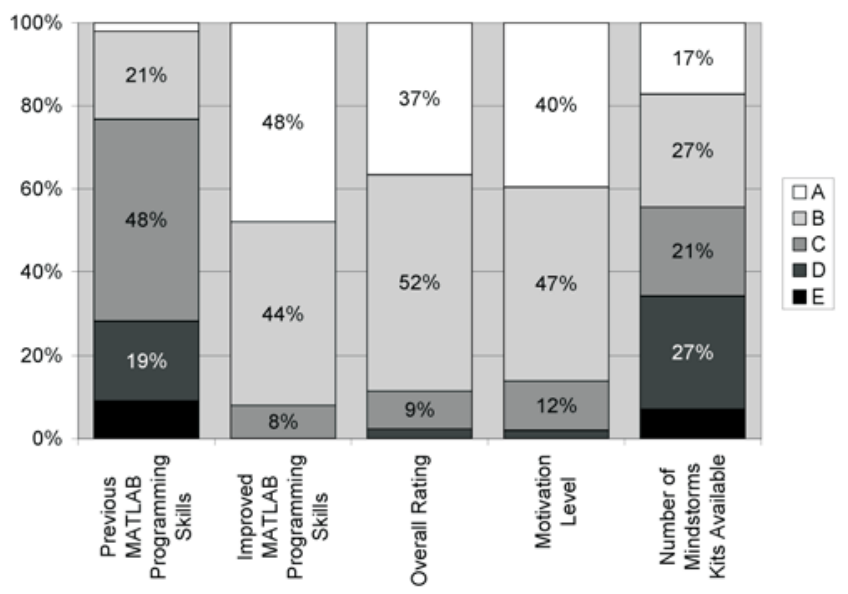

Fig. 11: Evaluation results (A: excellent, B: good, C: average, D: below average, $\mathrm{E}$ : inadequate)

The overall rating also shows an "excellent" or "good" validation of $89 \%$. In addition, almost every participant would join the lab again and approved the usage of Mindstorms applications also in other practical courses. Besides the motivation rate of Fig. 11, the students' motivation was expressed by their group dynamics, strong intention to compete with other groups by spending additional time on refining the robot algorithms after the official hours. The impact of peer learning within the groups of four was also observable, since the students shared resources and engaged in debates and critical reflections.

The question "Identify positive aspects of the lab" produced responses such as "Confrontation with problems which are not mentioned in the theory", "Theoretical foundations could be linked to practical problems, which led to a higher understanding level" and "Practical application with MATLAB" also showed that the proposed objectives were fulfilled.

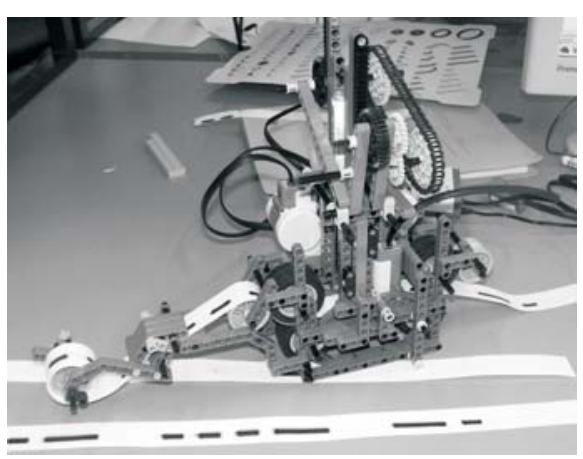

Fig. 12: Morse Encoder and Decoder Machine
Students' creativity and the variety of individual application ideas and robot constructions exceeded our expectations. The evaluation showed that $45 \%$ of the interviewed persons defined and implemented their own practical major tasks. Thus new and creative robots, like a 3-D environment scanner with target recognition, a bottle finder with fill-level-scanner, a vehicle with roadside recognition, a bowling robot, an autonomous parking vehicle, a sorting machine, a bar code and bar disk scanner, a Morse encoder/decoder (shown in Fig. 12), etc. were designed.

While many students argued that working with the hardware limitations was challenging, some participants criticized the occurrence of major Bluetooth latencies and the complex motor control, which limited the implementations of their applications. The limited number of Mindstorms kits was also criticized, especially during the basic exercises, in which one kit was shared by two teams of two students. Referring to this, Fig. 11 shows an "average" rating.

Overall, the laboratory work clearly showed that freshman students were highly motivated and encouraged to transfer mathematical principles to robotics while broadening their programming knowledge in MATLAB at the same time. Social skills like working in a team, managing the assigned tasks within a given time frame and cooperating with others to attain mutually beneficial goals were also supported by the laboratory concept. In addition, students who showed only minor creativity and productivity were encouraged to work on the predetermined and documented applications.

\section{Perspectives}

Based on this first experience and the evaluation results, some adjustments will be made for the next semester. The number of Mindstorms NXT education sets will be doubled in order to accelerate the execution time of the basic exercises and provide additional time for the major practical tasks. The RWTH - Mindstorms NXT Toolbox for MATLAB will support a USB interface to lower the probability of major packet losses using the Bluetooth channel, and additional high-level motor control functions will be implemented. Due to the great success of the project, this laboratory may also be integrated into the curriculum for Industrial Engineering.

\section{Conclusions}

This paper has presented an introductory course for freshmen in practical engineering. We have described a threefold learning concept which confirms mathematical basics, fosters MATLAB programming skills, and introduces students to real engineering problems, motivated by LEGO Mindstorms robots. A novel MATLAB toolbox, which provides direct computer-robot communication, has been presented and has been made publicly available as an open source project.

The sequential execution of basic and main practical tasks evoked a high student motivation, expressed by a wide range of creative and individual robotics applications and MATLAB solving algorithms. The inherent hardware limitations were challenging for most participants and led to intensive group dynamics and team work. The block course concept fulfilled the demanded practical engineering and MATLAB program- 
ming goals, which were also expressed by the students' final project presentations.

Extending this learning concept to other practical courses with interdisciplinary groups could broaden the students' professional skills and help their future careers.

\section{Project web page}

Further images, descriptions, and videos of the students' robots and inventions are available at the web page http://www.lfb.rwth-aachen.de/mindstorms.

The contact to the developer community and the free download of the "RWTH - Mindstorms NXT Toolbox" for MATLAB can be found at the project web page http://www.mindstorms.rwth-aachen.de.

\section{Acknowledgments}

The authors would like to thank Prof. Dr.-Ing. Til Aach, Institute of Imaging \& Computer Vision, and Prof. Dr.-Ing. Tobias G. Noll, Chair of Electrical Engineering and Computer Systems, RWTH Aachen University, who supervised this project.

The authors would also like to thank Rainer Schnitzler and Achim Knepper, who gave support to the project development and organization.

Finally, the authors gratefully acknowledge the work of Marian Walter and Axel Cordes, who tested and verified the toolbox and practical exercises.

\section{References}

[1] European Ministers of Education: Bologna Declaration, June 1999. [Online]. Available:

http://www.ond.vlaanderen.be/hogeronderwijs/bologna/ documents/

[2] Vallim, M., Farines, J.-M., Cury, J.: Practicing Engineering in a Freshman Introductory Course, IEEE Trans. Edu., Vol. 49 (2006), No. 1, p. 74-79, Feb. 2006.

[3] Director, S., Khosla, P., Rohrer, R., Rutenbar, R.: Reengineering the Curriculum: Design and Analysis of a New Undergraduate Electrical and Computer Engineering Degree at Carnegie Mellon University. Proc. IEEE, Vol. 83 (1995), No. 9, p. 1246-1269, Sep. 1995.

[4] Saint-Nom, R., Jacoby, D.: Building the first steps into SP Research. In Proc. IEEE Int. Conference on Acoustics, Speech, and Signal Processing (ICASSP), Vol. 5 (2005), p. 545-548.

[5] Hissey, T.: Education and Careers 2000. Enhanced Skills for Engineers. Proc. IEEE, Vol. 88 (2000), No. 8, p. 1367-1370, Aug. 2000.
[6] McClellan, J., Schafer, R., Yoder, M.: Signal Processing First. Prentice-Hall, Nov 2002.

[7] The MathWorks, MATLAB® . [Online]. Available: http://www.mathworks.com.

[8] LEGO MINDSTORMS® . [Online]. Available: http://www.mindstorms.com.

[9] LEGO ${ }^{\circledR}$ MINDSTORMS $®$ : Bluetooth developer kit. [Online]. Available: http://mindstorms.lego.com/Overview/NXTreme.aspx.

[10] McGoldrick, C., Huggard, M.: Peer Learning with Lego Mindstorms. In Proc. $34^{\text {th }}$ Frontiers in Education, Oct. 2004, p. S2F24-29.

[11] Patterson-McNeill, H., Binkerd, C. L.: Resources for Using Lego Mindstorms. J. Comput. Small Coll., Vol. 16 (2001), No. 3, p. 48-55.

[12] Hars, A., Ou, S.: Working for Free? - Motivations of Participating in Open Source Projects. Proc. $34^{\text {th }}$ Int. Conf. on System Sciences, p. 1-9, Jan. 2001.

[13] GNU General Public License. [Online]. Available: http://www.gnu.org/licenses/licenses.html.

[14] RWTH Aachen University: RWTH - Mindstorms NXT Toolbox for MATLAB. [Online]. Available: http://www.mindstorms.rwth-aachen.de.

Alexander Behrens

e-mail: behrens@lfb.rwth-aachen.de

Linus Atorf

e-mail: atorf@lfb.rwth-aachen.de

Institute of Imaging \& Computer Vision

Robert Schwann

e-mail:schwann@eecs.rwth-aachen.de

Chair of Electrical Engineering and Computer Systemsg

Johannes Ballé

e-mail: balle@ient.rwth-aachen.de

Chair of Institute of Communications Engineerin

Thomas Herold

e-mail: herold@iem.rwth-aachen.de

Institute of Electrical Machines

Aulis Telle

e-mail: telle@ind.rwth-aachen.de

Institute of Communication Systems and Data Processing

RWTH Aachen University

Templergraben 55

52062 Aachen, Germany 